\title{
Dispersion and induction interactions of graphene with nanostructures
}

\author{
John F. Dobson
}

(Dated: November 27, 2010)

\begin{abstract}
The unusual electronic bandstructure of graphene is shown to give it several novel properties beyond the much-studied transport behavior. The electron density response of a graphene sheet is studied here in some detail. It is thereby shown that the attractive forces between graphene and distant objects can be calculated analytically, and exhibit unusual power laws as a function of their separation $D$. A brief review is also given of recent progress with high-level numerical many-electron calculations involving graphene interactions near the equilibrium binding distance $D_{0}$.
\end{abstract}




\section{INTRODUCTION}

Most recent studies of graphene have focussed on its unusual electron transport properties, which are dominated by gapless electronic transitions between the $\pi_{z}$ and $\pi_{z}^{*}$ Bloch bands 22 . In this article we will focus on the interaction of graphene with other nano-objects and chemical species. We emphasize those properties that can be understood from the linear electronic response of graphene. Foremost among these is the notoriously tricky dispersion (van der Waals, vdW) interaction, whose correct description is vital if one wants to predict the cohesion and self-assembly of the great variety of proposed nanodevices based on graphene. The induction interaction is also if interest. For interacting objects at large distances, we shall find that the vdW and induction interactions, like the transport properties, are dominated by the gapless electronic transitions in graphene, so that a simple semianalytic approach is useful. This approach has led to the prediction [3], yet to be tested experimentally, of an unconventional power law $E=-K D^{-3}$ for the decay of the vdW interaction energy between parallel graphene sheets as a function of their (large) separation D. However when the second object is closer in, all transitions may become significant and more detailed modelling of graphenic electrons, beyond linear response, is also required. However even in these cases the linear response of graphene is useful since this is the basis of a very powerful high-level treatment of electronic correlations, the RPA (Random Phase Approximation) class of theories. These theories are well-known and essentially trivial for the uniform electron gas, but their numerical implementation for large periodic systems is very demanding, especially for the case of graphitic systems for reasons discussed below. Only now is the RPA beginning to produce the first reliable numbers for the van der Waals (dispersion) contribution to the cohesive properties of systems involving graphene [4]. Because of the need to go beyond the linear-dispersion $\pi$ - $\pi^{*}$ physics of graphene for the present applications, here we do not use the currently conventional analogy with massless chiral particles, but rather we employ the usual condensed matter approach with Bloch waves and plane-wave or Wannier-type expansions. 


\section{ELECTRONIC BANDSTRUCTURE AND LONG-WAVELENGTH DENSITY RESPONSE OF GRAPHENE}

\section{A Bloch waves in the Plane-Wave and Tight-Binding expansions.}

Eigenstates of a single independent electron moving in a periodic groundstate Kohn-Sham crystal potential (the $n t h$-band Bloch states) can be exactly represented by a plane wave expansion

$$
\psi_{\vec{k}}^{(n)}(\vec{r}, s)=\sum_{\vec{G}} c_{\vec{G}}^{(n)}(\vec{k}) \exp (i(\vec{k}+\vec{G}) \cdot \vec{r})
$$

or equivalently a Wannier (local-basis) represention using spatially localized functions $w$ :

$$
\psi_{\vec{k}}^{(n)}(\vec{r}, s)=N^{-1 / 2} \sum_{\vec{R}} \sum_{\ell=1}^{L} e_{\ell}^{(n)}(\vec{k}) w^{(n)}\left(\vec{r}-\vec{R}-\vec{x}_{\ell}\right) \exp \left(i \vec{k} \cdot\left(\vec{R}+\vec{x}_{\ell}\right)\right) .
$$

Here $\vec{R}$ is a direct lattice vector joining equivalent positions in different direct-space unit cells,

and $\{\vec{G}\}$ are the set of reciprocal basis vectors generated from the $\{\vec{R}\}$. The $\left\{\vec{x}_{\ell}, \ell=1,,, L\right\}$ are the $L$ positions within the direct unit cell, around which the Wannier functions $w$ are localized, measured relative to the direct cell origin. The Bloch function is correctly normalized because the Wannier amplitudes obey

$$
\sum_{\ell} e_{\ell}^{(n) *} e_{\ell}^{\left(n^{\prime}\right)}=\delta_{n n^{\prime}}
$$

where $n$ labels a complex of contiguous bands (e.g. the $\pi_{z}, \pi_{z}^{*}$ band complex in graphene).

\section{B Bloch bandstructure of graphene}

In graphene [5] the $2 \mathrm{D}$ direct cell is hexagonal with direct lattice vectors

$$
\vec{a}_{(1)}=\frac{a}{2}(\sqrt{3}, 1), \quad \vec{a}_{(2)}=\frac{a}{2}(\sqrt{3},-1)
$$

and contains two carbon atoms at the bottom corners of the hexagonal conventional cell, with positions

$$
\vec{x}_{1}=(0,0), \vec{x}_{2}=a(0,1 / \sqrt{3})
$$


relative to the cell origin at the bottom left corner. Here $a \approx 0.246 \mathrm{~nm}$ is the graphene lattice spacing. We will assume that the Wannier orbitals are centred on the atoms, which is an allowed choice, though Wannier functions have a phase ambiguity that can be exploited to increase their localization, and the most localized Wannier functions are actually centred at the middle of the the $\pi_{z}$ bonds joining atoms.

For a general periodic system in its groundstate, the exact groundstate density is obtained from a sum $n(\vec{r})=\sum_{\vec{k}, s \text { occ }}\left|\psi_{\vec{k}, s}(r)\right|^{2}$ where the Bloch functions $\psi_{\vec{k}, s}$ are the KohnSham eigenfunctions satisfying the 1-body Schroedinger equation in the (periodic) groundstate Kohn Sham potential $V^{K S}(\vec{r})$. Quite realistic values of $V^{K S}(\vec{r})$ can be obtained from three-dimensional periodic plane-wave codes such as ABINIT [6],[7] or VASP [8],[9], which implement the self-consistent Local Densty Approximation (LDA) or Generalized Gradient Approximation (GGA) for the many-electron interactions.. For the case of graphene, this can in principle be done in such 3D codes by treating a highly stretched graphite lattice, so that each graphene sheet is widely separated from the others.

For some of what follows we do not need such a complete description, but rather we will be concerned mainly with the $\pi_{z}$ Bloch bands whose localized orbitals $w$ have their lobes pointing in the $\pm z$ directions perpendicular to the sheet, forming $\pi$ clouds to either side of the plane of carbon atoms. A simple analytic approximation for these $\pi_{z}$ Bloch states and their energy eigenvalues was obtained by Wallace as long ago as 1947 [10]: see also Ch 2 of $[5]$.

$$
\psi^{\text {valence(conduction) }}(\vec{k}, \vec{r})=\sum_{\vec{R}}\left(e_{1}(\vec{k}) w^{\left(\pi_{z}\right)}\left(\vec{r}-\vec{R}-\vec{x}_{1}\right)+e_{2}(\vec{k}) w^{\left(\pi_{z}\right)}\left(\vec{r}-\vec{R}-\vec{x}_{2}\right)\right) e^{i \vec{k} . \vec{R}}
$$

Here $w(r)$ is a localized function, and the approach can be made formally exact for a group of bands if one uses the Wannier function for the $w_{i}$. By demanding that the expectation of the 1-electron Hamiltion $\hat{H}$ be optimised with respect to the mixing coefficients $e_{1}$ and $e_{2}$ subject to normalization, we obtain a $2 \times 2$ eigenvalue equation

$$
\sum_{\ell^{\prime}=1}^{2}\left(H_{\ell \ell^{\prime}}(\vec{k})-S_{\ell \ell^{\prime}}(\vec{k})\right) e_{\ell^{\prime}}=E e_{\ell} .
$$


where $H$ and $S$ give the Hamiltonian and overlap matrix elements respectively, i.e.

$$
\begin{aligned}
& H_{\ell \ell^{\prime}}(\vec{k})=\sum_{\vec{R}} \int e^{i \vec{k} \cdot \vec{R}} w^{\left(\pi_{z}\right) *}\left(\vec{r}-\vec{x}_{\ell}\right) \hat{H} w^{\left(\pi_{z}\right)}\left(\vec{r}-\vec{x}_{\ell^{\prime}}-\vec{R}\right) d \vec{r}, \\
& S_{\ell \ell^{\prime}}(\vec{k})=\sum_{\vec{R}} e^{i \vec{k} \cdot \vec{R}} \int w^{\left(\pi_{z}\right) *}\left(\vec{r}-\vec{x}_{\ell}\right) w^{\left(\pi_{z}\right)}\left(\vec{r}-\vec{x}_{\ell^{\prime}}-\vec{R}\right) d \vec{r} .
\end{aligned}
$$

Here, following Wallace we make the tight binding approximation, neglecting all but the lowest nontrivial overlap terms in Eq.(6), involving on-site and nearest-neighbor terms. Then for graphene Eq. (5) becomes

$$
\left(\begin{array}{cc}
\varepsilon-E & H_{21}^{*}(\vec{k}) \\
H_{21}(\vec{k}) & \varepsilon-E
\end{array}\right)\left(\begin{array}{l}
e_{1} \\
e_{2}
\end{array}\right)=\left(\begin{array}{l}
0 \\
0
\end{array}\right)
$$

where $\varepsilon=H_{11}=H_{22}=\int w^{\left(\pi_{z}\right) *}(\vec{r}) \hat{H} w^{\left(\pi_{z}\right)}(\vec{r}) d \vec{r}, S_{\ell \ell^{\prime}}=\int w^{\left(\pi_{z}\right)}\left(\vec{r}-\vec{x}_{\ell}\right) w^{\left(\pi_{z}\right)}\left(\vec{r}-\vec{x}_{\ell^{\prime}}\right) d \vec{r} \approx \delta_{\ell \ell^{\prime}}$ and

$$
H_{21}(\vec{k})=t\left(1+e^{i \vec{k} \cdot \vec{a}_{1}}+e^{i \vec{k} \cdot \vec{a}_{2}}\right)=H_{12}^{*}(\vec{k})
$$

Here $t$ is the hamiltonian matrix element between two neighboring localised orbitals, $t=$ $\int w^{\left(\pi_{z}\right)}\left(\vec{r}-\vec{x}_{1}\right) \hat{H} w^{\left(\pi_{z}\right)}\left(\vec{r}-\vec{x}_{2}\right) d \vec{r}$.

The solutions of Eq. (7) are

$$
\begin{gathered}
E^{\text {conduction(valence) }}(\vec{k})=\varepsilon \pm\left|H_{21}(\vec{k})\right| \\
\left|H_{21}(\vec{k})\right|=t \sqrt{1+4 \cos \left(\frac{k_{y} a}{2}\right) \cos \left(\frac{\sqrt{3}}{2} k_{x} a\right)+4 \cos ^{2}\left(\frac{k_{y} a}{2}\right)} \\
\frac{e_{2}^{(\pi z)}}{e_{1}^{(\pi z)}}=-\frac{H_{21}(\vec{k})}{\varepsilon-E_{\vec{k}}}= \pm \frac{H_{21}(\vec{k})}{\left|H_{21}(\vec{k})\right|}=s a y \pm e^{i \phi(\vec{k})} \text { for conduction/valence respectively }(11) \\
e_{1}^{(\pi z)} .=\frac{1}{\sqrt{2}}, e_{2}^{(\pi z)}= \pm \frac{1}{\sqrt{2}} e^{i \phi_{k}}
\end{gathered}
$$

The conduction and valence $\pi_{z}$ Bloch functions are then

$$
\psi^{\text {conduction(valence) }}=\sum_{\vec{R}} e^{i \vec{k} . \vec{R}}\left(w(\vec{r}-\vec{R}) \pm \frac{H_{21}(\vec{k})}{\left|H_{21}(\vec{k})\right|} w\left(\vec{r}-\vec{x}_{2}-\vec{R}\right)\right)
$$


where we have chosen the direct cell so that $\vec{x}_{1}=\overrightarrow{0}$, as in (3), and we have dropped the band lanel $n=\pi_{z}$. Note that the coupling Eq. (8) vanishes at the two "Dirac points"

$$
K_{1(2)}=\frac{2 \pi}{\sqrt{3} a} \vec{i} \pm \frac{2 \pi}{3 a} \vec{j}
$$

i.e. $H_{12}\left(\vec{K}_{1(2)}\right)=0$. The two solutions Eq. (9) therefore describe conduction $\left(\pi_{z}^{*}\right)$ and valence $\left(\pi_{z}\right)$ bands that touch (have the same energy) at the Dirac points. This is the origin of the gapless semimetallic behavior of undoped graphene at $T=0 K$, since for that case the $\pi_{z}$.band is full and the $\pi_{z}^{*}$ band is empty.

Near either Dirac point $\vec{K}=\vec{K}_{1}$ or $\vec{K}_{2}$ we write $\vec{k}=\vec{K}+\vec{p}$ where $|\vec{p}|<<|\vec{K}|$. There the coupling Hamiltonian vanishes linearly with $\vec{p}$

$$
\begin{aligned}
H_{21}(\vec{k}) / t & =1+e^{4 \pi i / 3}\left(\cos \vec{p} \cdot \vec{a}_{1}+i \sin \vec{p} \cdot \vec{a}_{1}\right)+e^{2 \pi i / 3}\left(\cos \vec{p} \cdot \vec{a}_{2}+i \sin \vec{p} \cdot \vec{a}_{2}\right), \quad \vec{k}=\vec{K} \overrightarrow{+} \vec{p} \\
& =-i \frac{\sqrt{3} a}{2}\left(p_{x}+i p_{y}\right)+O\left(p^{2}\right) \text { for small }|\vec{p}|<<a^{-1}
\end{aligned}
$$

Then the $3 \mathrm{D}$ bandstructure diagram ( $E$ plotted vertically versus $p_{x}, p_{y}$ in the horizonal plane) is a cone:

$$
E_{K+p}^{\text {conduction } / \text { valence }} \approx \varepsilon \pm \hbar v_{0}|p|, v_{0}=\frac{\sqrt{3}}{2 \hbar} t a
$$

Detailed calculations within the Local Density Approximation (via VASP software) suggest that

$$
v_{0} \approx 1 \times 10^{6} \mathrm{~m} / \mathrm{s}
$$

and they also show that the energy $E_{k}$ remains rather linear in $|\vec{p}|$ at energies up to more than $2 \mathrm{eV}$ above or below the Dirac points, eventually turning over to create finite $\pi_{z} \pi_{z}^{*}$ bandwidths (see also [5]).

\section{Bare (Kohn-Sham) linear response of graphene}

First consider an arbitrary $N$-electron system, initially in its paramagnetic groundstate described by a set of doubly occupied ( $\uparrow$ and $\downarrow$ ) Kohn-Sham orbitals $\psi_{i}(\vec{r})$ that yield the groundstate electron density, and that satisfy $\left(-\hbar^{2}(2 m)^{-1} \nabla^{2}+V_{K S}(\vec{r})\right) \psi_{i}(\vec{r})=\varepsilon_{i} \psi_{i}(\vec{r})$ with 
a common Kohn-Sham potential $V_{K S}(\vec{r})$. Then apply to the electrons, in addition to $V^{K S}(r)$, a small potential energy perturbation $\delta v$ in the form

$$
\delta v=e^{u t} \vec{v}(r)
$$

whose time dependence corresponds to an imaginary frequency;

$$
\omega=i u
$$

In calculating the electronic response to this potential, we start by ignoring the electronelectron interaction, yielding the so-called Kohn Sham or bare response. (Later we include the electron-electron interaction via an additional term in $\delta v$, leading to theories such as the Random Phase Approximation). Straightforward first order time dependent perturbation theory yields the perturbation $\delta \psi_{\vec{k}}$ to each 1-electron state, and the density response is

$$
\begin{aligned}
\delta n(\vec{r}, t) & =\sum_{\vec{k} \text { occ }}\left(\psi_{\vec{k}}^{(0)}(\vec{r}) \delta \psi_{k}^{*}(\vec{r}, t)+\psi_{\vec{k}}^{(0) *}(\vec{r}) \delta \psi_{\vec{k}}(\vec{r}, t)\right) \\
& =e^{u t} \int \chi_{K S}\left(\vec{r}, \vec{r}^{\prime}, i u\right) v\left(\vec{r}^{\prime}\right) d \vec{r}
\end{aligned}
$$

where

$$
\chi_{K S}\left(\vec{r}, \vec{r}^{\prime}, i u\right)=2 \sum_{i j} \frac{f_{i}-f_{j}}{\varepsilon_{j}-\varepsilon_{i}-i \hbar u} \psi_{i}^{*}(\vec{r}) \psi_{j}^{*}\left(\vec{r}^{\prime}\right) \psi_{i}\left(\vec{r}^{\prime}\right) \psi_{j}(\vec{r})
$$

and where $f_{i}$ is the Fermi occupation factor, and the factor of 2 is for spins.

For an infinite 3D periodic array of atoms or molecules we consider an external potential of the quasiperiodic form

$$
-e \phi^{e x t}=v^{e x t}(\vec{r}, t)=v_{\vec{q}, \vec{G}}^{e x t} \exp (i(\vec{q}+\vec{G}) \cdot \vec{r}) \exp (u t)
$$

where the wavevector $\vec{q}$ is restricted to the first Brillouin Zone but the reciprocal lattice vector $\vec{G}$ allows a perturbation of arbitrary wavevector to be described. We find

$$
\delta n(r, t)=e^{u t} \sum_{\vec{G}^{\prime}} e^{i\left(\vec{q}+\vec{G}^{\prime}\right) \cdot \vec{r}} \chi_{K S n n^{\prime}}\left(\vec{q}, \vec{G}, \vec{G}^{\prime}, i u\right) v_{\vec{q}, \vec{G}}^{e x t}
$$


where the periodic density-density response is obtained by putting Bloch waves $\psi_{\vec{k}}$ into Eq. (15) is:

$$
\begin{aligned}
\chi_{K S n n^{\prime}}\left(\vec{q}, \vec{G}, \vec{G}^{\prime}, i u\right) & =2 \sum_{\vec{p}, \vec{p}^{\prime}} \frac{f_{\vec{p} n}-f_{\vec{p}^{\prime} n^{\prime}}}{\varepsilon_{\vec{p}}^{(n)}-\varepsilon_{\vec{p}^{\prime}}^{\left(n^{\prime}\right)}-i \hbar u} \delta_{\vec{p}^{\prime}-\vec{p}-\vec{q}} \\
\times & <\vec{p} n\left|V^{-1 / 2} e^{-i(\vec{q}+\vec{G}) \cdot \vec{r}}\right| \vec{p}^{\prime} n^{\prime}><\vec{p}^{\prime} n^{\prime}\left|V^{-1 / 2} e^{i\left(\vec{q}+\vec{G}^{\prime}\right) \cdot \vec{r}^{\prime}}\right| \vec{p} n>.
\end{aligned}
$$

Here $\langle\vec{p} n \mid r\rangle=\psi_{k}^{(n)}(\vec{r}), \vec{p}$ is summed (integrated) over the 3D Brillouin zone and $n$ and $n^{\prime}$ are summed over all Bloch bands. Eq. (16), with the plane wave representation Eq. (1), is the basis for accurate (but very time-consuming) calculation of $\chi_{K S}$ within plane-wave codes such as ABINIT [6],[7] or VASP [8],[9]. To analyze 2D graphene via this approach, one makes a very widely spaced periodic array of parallel graphene sheets (stretched graphite, "straphite"), which is numerically difficult in practice. For semianalytic evaluation, however, we consider a single graphene sheet in the following Sections.

\section{Semi-analytic $\chi_{K S}$ for graphene in the tight binding approach}

Evaluation of $\chi_{K S}$ for 3D crystals within the TB approximation was analyzed by Hanke [11], and the approach here is somewhat similar, though for a $2 \mathrm{D}$ system with a real space $\left(z, z^{\prime}\right)$ description in the direction perpendicular to the plane, and generalized to more than one atom per unit cell. Our explicit analytic evaluation will be limited to that part of the response that comes from mixing the $\pi_{z}$ and $\pi_{z}^{*}$ electronic states. See also [12]. We define $\vec{q}_{\|}=q_{x} \hat{x}+q_{y} \hat{y}$ to be the component of wavenumber parallel to the graphene plane. Because of their gapless nature, we shall find that the $\pi_{z}-\pi_{z}^{*}$ transitions cause a strong $q_{\|}$and $u$ dependence of the polarizability $\alpha_{\|} \equiv q_{\|}^{-2} \chi$ for small $q_{\|}$and $u$ values, and their contribution to the response will therefore tend to dominate the interactions with very distant objects. For objects at intermediate separations, the other transitions cannot always be neglected, but they are gapped and make a polarizability contribution that depends smoothly on $q_{\|}$. We can therefore treat these in a Lifshitz-like [13] model with local $\left(\overrightarrow{q^{-}}\right.$ independent) polarizabilities $\alpha_{\|}(i u)$ and $\alpha_{\perp}(i u)$ parallel and perpendicular to the graphene 
plane, to be discussed elsewhere.

For graphene we consider the response to a potential varying as a plane-wave in the $\mathrm{x}$ and y directions parallel to the graphene plane:

$$
\delta v=e^{u t} e^{i(\vec{q}+\vec{G}) \cdot \vec{r}} \delta v(z)
$$

where $\vec{q} \equiv q_{\|}$is now a $2 \mathrm{D}$ vector lying in the $2 \mathrm{D}$ graphene Brillouin zone, and $\vec{G}$ is a $2 \mathrm{D}$ reciprocal lattice vector. The density resonse is of form

$$
\delta n(\vec{r}, t)=e^{u t} \delta n\left(\vec{r}_{\|}, z\right)=e^{u t} \sum_{\vec{G}^{\prime}} e^{i\left(\vec{q}+\vec{G}^{\prime}\right) \cdot \vec{r}_{\|}} \int \chi_{K S}\left(\vec{q}, \vec{G}, \vec{G}^{\prime}, z, z^{\prime}, i u\right) \delta v\left(z^{\prime}\right) d z^{\prime}
$$

where we have used the response Eq. (15) projected onto 2D plane waves:

$$
\begin{aligned}
& \chi_{K S}\left(\vec{q}, \vec{G}, \vec{G}^{\prime}, z, z^{\prime}, \omega\right)=<\vec{q}+\vec{G}\left|\chi_{K S}\right| \vec{q}+\vec{G}^{\prime}> \\
& =\int d^{2} \vec{r}_{\|} d^{2} \vec{r}_{\|}^{\prime} \frac{e^{-i(\vec{q}+\vec{G}) \cdot \vec{r}_{\|}}}{A^{1 / 2}} \frac{e^{i\left(\vec{q}+\vec{G}^{\prime}\right) \cdot \vec{r}_{\|}}}{A^{1 / 2}} \chi_{K S}\left(\vec{r}, \vec{r}^{\prime}, \omega\right) \\
& =2 \sum_{\vec{p} \in B Z, n, \vec{p}^{\prime} \in B Z, n^{\prime}} \frac{f_{\vec{p} n}-f_{\vec{p}^{\prime} n^{\prime}}}{\varepsilon_{\vec{p}}^{(n)}-\varepsilon_{\vec{p}^{\prime}}^{\left(n^{\prime}\right)}-\hbar \omega}<\vec{p} n, z\left|A^{-1 / 2} e^{-i(\vec{q}+\vec{G}) \cdot \vec{r}_{\|}}\right| \vec{p}^{\prime} n^{\prime}, z> \\
& \times<\vec{p}^{\prime} n^{\prime}, z^{\prime}\left|A^{-1 / 2} e^{\left.i\left(\vec{q}+\vec{G}^{\prime}\right) \cdot \vec{r}^{\prime}\right|^{\prime}}\right| \vec{p} n, z^{\prime}>.
\end{aligned}
$$

Here the matrix elements represent integration over $\vec{r}_{\|}$only (i.e. in the the $\mathrm{x}$ and $\mathrm{y}$ directions). Here the bands $n, n^{\prime}$ are selected from $\pi_{z}$ and $\pi_{z}^{*}$. where $\left\langle\vec{k} n, z \mid \vec{r}_{\|}\right\rangle=\psi_{\vec{k}}^{(n)}\left(\vec{r}_{\|}, z\right)$ is a graphene $\pi_{z}$ Bloch state and $\vec{k}$ is a $2 \mathrm{D}$ vector lying in the 2D Brillouin Zone (BZ). To evaluate the plane wave matrix elements between the Bloch states of a single $2 \mathrm{D}$ graphene sheet we expand them in localized $p_{z}$ functions $w(\vec{r})$ as in Eq. (2), with the sum over $\vec{R}$ now carried out on the 2D graphene lattice, and $\vec{q}$ and $G$ are now two -dimensional vectors. The matrix element becomes

$$
\begin{aligned}
& <\vec{p} n, z\left|A^{-1 / 2} e^{-i(\vec{q}+\vec{G}) \cdot \vec{r}_{\|}}\right| \vec{p}^{\prime} n^{\prime}, z>A^{-1 / 2}\left\langle\psi_{\overrightarrow{p^{\prime}}}^{\text {valence }}(\vec{r} \|, z)\left|\exp \left(i(\vec{q}+\vec{G}) \cdot \vec{r}_{\|}\right)\right| \psi_{\vec{p}}^{\text {cond }}\left(\vec{r}_{\|}, z\right)\right\rangle \\
& =A^{-1 / 2}\left(\sum_{\vec{G}^{\prime \prime}} \delta_{\vec{p}^{\prime}-\vec{p}-\vec{q}-\vec{G}, \vec{G}^{\prime \prime}}\right) \sum_{\ell, \ell^{\prime}=1}^{2} e_{\ell}^{(n) *}(\vec{p}) e_{\ell^{\prime}}^{\left(n^{\prime}\right)}(\vec{p}+\vec{q}) \sum_{\vec{X}} e^{i(\vec{p}+\vec{q}) \cdot \vec{X}} S_{\ell, \ell^{\prime}, \vec{X}}^{\left(n, n^{\prime}\right)}(\vec{q}+\vec{G}, z)
\end{aligned}
$$

where $A$ is the (quasi-infinite) area of the graphene sheet, $\vec{X}$ is a $2 \mathrm{D}$ direct lattice vector and the $\{\vec{G}\}$ are the $2 \mathrm{D}$ reciprocal lattice vectors. Denoting $\left(\ell, \ell^{\prime}, \vec{X}\right)$ by $\mu$ and $\left(\ell^{\prime \prime}, \ell^{\prime \prime \prime}, \vec{X}^{\prime \prime}\right)$ by $\mu^{\prime \prime}$ and putting the above into (17) we obtain the KS response 


$$
\begin{aligned}
& \text { Note citation numbering is out by } 1 \\
& \chi_{K S}\left(\vec{q}, \vec{G}, \vec{G}^{\prime}, z, z^{\prime}, \omega\right)=\sum_{\mu, \mu^{\prime \prime}, n, n^{\prime}} J_{\mu, \mu^{\prime \prime}}^{\left(n, n^{\prime}\right)}(\vec{q}, \omega) S_{\mu}^{\left(n . n^{\prime}\right)}(\vec{q}+\vec{G}, z) S_{\mu^{\prime \prime}}^{\left(n . n^{\prime}\right) *}\left(\vec{q}+\vec{G}^{\prime}, z^{\prime}\right) \\
& J_{\mu, \mu^{\prime \prime}}^{\left(n, n^{\prime}\right)}(\vec{q}, \omega)=A^{-1} \sum_{\vec{k} \in B Z} \frac{f_{\vec{k}}^{(n)}-f_{\vec{k}+\vec{q}}^{\left(n^{\prime}\right)}}{\varepsilon_{\vec{k}}^{(n)}-\varepsilon_{\vec{k}+\vec{q}}^{\left(n^{\prime}\right)}-\hbar \omega} e_{\ell}^{(n) *}(\vec{k}) e_{\ell^{\prime}}^{\left(n^{\prime}\right)}(\vec{k}+\vec{q}) e_{\ell^{\prime \prime}}^{(n)}(\vec{k}) e_{\ell^{\prime \prime \prime}}^{\left(n^{\prime}\right) *}(\vec{k}+\vec{q}) e^{-i(\vec{k}+\vec{q}) \cdot\left(\vec{X}-\vec{X}^{\prime}\right)}
\end{aligned}
$$

Here $\varepsilon_{\vec{k}}^{(n)}, e$ and $f$ are defined initially for $\vec{k}$ in the 2D Brillouin zone but are continued outside the zone with reciprocal lattice periodicity, and

$$
\begin{aligned}
S_{\mu}^{\left(n, n^{\prime}\right)} & \equiv S_{\ell, \ell^{\prime}, \vec{X}}^{\left(n, n^{\prime}\right)}(\vec{q}+\vec{G}, z) \\
& \left.=\int d^{2} x_{\|} x e^{-i(\vec{q}+\vec{G}) \cdot \vec{x}^{(n) *}} w^{\left(\vec{x}_{\|}\right.}+z \hat{z}-\vec{x}_{\ell}\right) w^{\left(n^{\prime}\right)}\left(\vec{x}_{\|}+z \hat{z}-\vec{x}_{\ell^{\prime}}+\vec{X}\right) \\
& =e^{-i(\vec{q}+\vec{G}) \cdot\left(\vec{x}_{\ell}-\vec{x}_{\ell^{\prime}}\right)} \int d^{2} y_{\|} x e^{-i(\vec{q}+\vec{G}) \cdot \vec{y}_{\|}} w^{(n) *}\left(\vec{y}_{\|}+z \hat{z}\right) w^{\left(n^{\prime}\right)}\left(\vec{y}_{\|}+z \hat{z}+\vec{X}+\vec{x}_{\ell}-\vec{x}_{\ell^{\prime}}(21)\right.
\end{aligned}
$$

Eq. (18) generalizes the work of Hanke [11] to the case of multiple atoms in the unit cell, so that the Wannier functions $w$ may be localized at different space points $\vec{x}_{\ell}$ inside the direct $2 \mathrm{D}$ unit cell.

For the response due to $\pi_{z}-\pi_{z}^{*}$ transitions at $T=0 K,(18)$ simplifies considerably. The contiguous $\pi_{z}$ and $\pi_{z}^{*}$ (gapless valence and conduction) bands are described by a single localized function $w$ repeated at the points $\vec{x}_{1}=0, \vec{x}_{2}=\frac{1}{2}\left(\vec{a}_{(1)}+\vec{a}_{(2)}\right)$ relative to the direct cell origin. Thus we can omit the $\left(n . n^{\prime}\right)$ indices on $w$ and $S$. The $\pi_{z}$ band is full and the $\pi_{z}^{*}$ band is empty, so $f=1$ or 0 . Then $\chi_{K S}$ contains nonzero "on-site" $\left(\ell=\ell^{\prime}, X=\right.$ $\left.0, \ell^{\prime \prime}=\ell^{\prime \prime \prime}, \vec{X}^{\prime}=\overrightarrow{0}\right)$ contributions and one may also want to inclulde"nearest-neighbor" contributions involving overlap integrals $S$ between adjacent localised functions $w$.

\section{E On-site contribution to $\chi_{K S}$}

Consider imaginary frequency $\omega=i u$ and keep only the "on-site" terms in Eq. (18) The resulting "on-site" response can be written

$$
\chi_{K S}^{o n-s i t e}\left(\vec{q}, \vec{G}, \vec{G}^{\prime}, z, z^{\prime}, i u\right)=\left(\sum_{\mu, \mu^{\prime} n, n^{\prime}} J_{\mu, \mu^{\prime \prime}}^{\left(n, n^{\prime}\right)}(\vec{q}, i u)\right) S_{0}(\vec{q}+\vec{G}, z) S_{0}^{*}\left(\vec{q}+\vec{G}^{\prime}, z^{\prime}\right)
$$




$$
S_{0}(\vec{q}+\vec{G}, z)=\int d^{2} y_{\|} x e^{-i(\vec{q}+\vec{G}) \cdot \vec{y}_{\|}} w^{*}\left(\vec{y}_{\|}+z \hat{z}\right) w\left(\vec{y}_{\|}+z \hat{z}\right)
$$

Now

$$
\sum_{n^{\prime} n^{\prime}, \mu . \mu^{\prime \prime}} J_{\mu, \mu^{\prime \prime}}^{\left(n, n^{\prime}\right)}(\vec{q}, i u)=\sum_{\ell, \ell^{\prime \prime}}\left(J_{\ell \ell, \vec{X}=0: \ell^{\prime \prime} \ell^{\prime \prime} X^{\prime}=\overrightarrow{0},}^{(v a l, c o n d}+J_{\ell \ell, \vec{X}=0: \ell^{\prime \prime} \ell^{\prime \prime} X^{\prime}=\overrightarrow{0},}^{(c o n d, v a l)}\right)
$$

and using Eq. (9) we obtain

$$
\begin{aligned}
\sum_{n^{\prime} n^{\prime}, \mu . \mu^{\prime \prime}} J_{\mu, \mu^{\prime \prime}}^{\left(n, n^{\prime}\right)}(\vec{q}, i u)= & A^{-1} \sum_{\vec{k} \in B Z} \frac{+1}{-\left|H_{12}(\vec{k})\right|-\left|H_{12}(\vec{k}+\vec{q})\right|-i \hbar u} \\
& \times\left|\left(\frac{1}{\sqrt{2}}\right)^{*}\left(\frac{1}{\sqrt{2}}\right)+\left(-\frac{1}{\sqrt{2}} e^{i \phi(\vec{k})}\right)^{*}\left(\frac{e^{i \phi(\vec{k}+\vec{q})}}{\sqrt{2}}\right)\right|^{2} \\
& +A^{-1} \sum_{\vec{k} \in B Z} \frac{-1}{\left|H_{12}(\vec{k})\right|+\left|H_{12}(\vec{k}+\vec{q})\right|-i \hbar u} \\
\times & \left|\left(\frac{1}{\sqrt{2}}\right)^{*}\left(\frac{1}{\sqrt{2}}\right)+\left(\frac{e^{i \phi(\vec{k})}}{\sqrt{2}}\right)^{*}\left(-\frac{e^{i \phi(\vec{k}+\vec{q})}}{\sqrt{2}}\right)\right|^{2} \\
\sum_{n^{\prime} n^{\prime}, \mu . \mu^{\prime \prime}} J_{\mu, \mu^{\prime \prime}}^{\left(n, n^{\prime}\right)}(\vec{q}, i u) & =A^{-1} \sum_{\vec{k} \in B Z} \frac{-2 \bar{\epsilon}}{\left.\bar{\epsilon}^{2}+(\hbar u)^{2}\right)} \frac{1}{4}\left|1-e^{i \Delta \phi}\right|^{2} \\
& =\frac{-1}{(2 \pi)^{2}} \int_{B Z} \frac{2 \bar{\epsilon}}{\left.\bar{\epsilon}^{2}+(\hbar u)^{2}\right)}(1-\cos \Delta \phi) d^{2} k
\end{aligned}
$$

where $\bar{\epsilon},=\left|H_{12}(\vec{k})\right|+\left|H_{12}(\vec{k}+\vec{q})\right|$ and $\Delta \phi=\phi(\vec{k}+\vec{q})-\phi(\vec{k})$. Then

$$
\chi_{K S}^{\text {on-site }}\left(\vec{q}, \vec{G}, \vec{G}^{\prime}, z, z^{\prime}, i u\right)=S_{0}(\vec{q}+\vec{G}, z) S_{0}^{*}\left(\vec{q}+\vec{G}^{\prime}, z^{\prime}\right) \bar{\chi}_{K S}(\vec{q}, i u)
$$

where

$$
\bar{\chi}_{K S}(\vec{q}, i u)=\frac{-1}{(2 \pi)^{2}} \int_{B Z} \frac{2 \bar{\epsilon}}{\left.\bar{\epsilon}^{2}+(\hbar u)^{2}\right)}(1-\cos \Delta \phi) d^{2} k
$$

and where $S_{0}$ is given by Eq. (23). The terms neglected in the on-site approximation Eq. (24) involve nearest neighbor overlap integrals (from $S$ ) form

$$
S_{\ell \ell^{\prime} \vec{X}}(\vec{q}+\vec{G}, z)=e^{i \theta} \int d^{2} y_{\|} x e^{-i(\vec{q}+\vec{G}) \cdot \vec{y}_{\|}} w^{(n) *}\left(\vec{y}_{\|}+z \hat{z}\right) w^{\left(n^{\prime}\right)}\left(\vec{y}_{\|}+z \hat{z}+\vec{X}+\vec{x}_{\ell}-\vec{x}_{\ell^{\prime}}\right)
$$




\section{F On-site approximation is exact for the "head" of the layer-integrated $\chi_{K S}$.}

The $\vec{G}=\vec{G}^{\prime}=\overrightarrow{0}$ term of the KS response is sometimes termed the "head" of the response matrix. For interaction with a distant object we typically need only head of the layer-integrated ( $z, z^{\prime}$ integrated) response

$$
\chi_{K S}\left(\vec{q}, \vec{G}=0, \vec{G}^{\prime}=\overrightarrow{0}, i u\right)=\iint \chi_{K S}\left(\vec{q}, \overrightarrow{0}, \overrightarrow{0}^{\prime}, z, z^{\prime}, i u\right) d z d z^{\prime}
$$

In this case the overlap integrals $S$ in Eq. (22) are z-integrated. As $q \rightarrow 0$ we obtain

$$
\bar{S}_{\ell \ell^{\prime} X} \equiv \int S(\vec{q} \rightarrow 0, \vec{G}=0, z) d z=\int d^{3} y_{\|} w^{(n) *}(\vec{y}) w^{\left(n^{\prime}\right)}\left(\vec{y}_{\|}+\vec{X}+\vec{x}_{\ell}-\vec{x}_{\ell^{\prime}}\right)=\delta_{\vec{X} \overrightarrow{0}} \delta_{\ell \ell^{\prime}}
$$

because of the orthonormality of the Wannier functions. Thus the "head" $\left(\vec{G}=\vec{G}^{\prime}=\overrightarrow{0}\right.$ element) of the z-integrated long-wavelength bare response $\chi_{K S}\left(\vec{q} \rightarrow \overrightarrow{0}, \vec{G}=0, \vec{G}^{\prime}=\overrightarrow{0}, i u\right)$ is given exactly by the reduced response Eq. (25). That is

$$
\chi_{K S}\left(\vec{q} \rightarrow \overrightarrow{0}, \vec{G}=0, \vec{G}^{\prime}=\overrightarrow{0}, i u\right)=\bar{\chi}_{K S}(\vec{q} \rightarrow \overrightarrow{0}, i u)
$$

where $\bar{\chi}_{K S}(\vec{q}, i u)$ is the reduced on-site response given in Eq. (25). For all other elements with $\vec{G}, \vec{G}^{\prime}$ not both zero, Eq. (24) with Eq. (25) remains an "on-site" approximation, even when $z, z^{\prime}$ integration is performed.. Note also that the layer-integrated form is not sufficient to study the screening interaction amongst electrons in the same layer: for that pupose we retain the $z, z^{\prime}$ variables.

\section{G Evaluating the singular contribution to the reduced response}

The linearity of the Bloch dispersion relation for graphene near a Dirac point $\vec{K}$ permits analytic evaluation of the most singular part of the reduced response $\bar{\chi}_{K S}^{\sin g}(\vec{q} \rightarrow \overrightarrow{0}, i u)$, which comes from these regions. Writing $\vec{k}=\vec{K}+\vec{p}$ we find from Eq. (13) that, for small $p$

$$
\begin{aligned}
\cos \Delta \phi & =\operatorname{Re} e^{i \Delta \phi}=\operatorname{Re} \frac{\left(p_{x}+i p_{y}\right)}{|\vec{p}|} \frac{p_{x}^{\prime}-i p_{y}^{\prime}}{|\vec{p}|}=\frac{\vec{p} \cdot \vec{p}^{\prime}}{|\vec{p}||\vec{p}|} \text { where } \vec{p}^{\prime}=\vec{p}+\vec{q} \\
\left|H_{12}(\vec{K}+\vec{p})\right| & =\hbar v_{0}|\vec{p}|, \quad \bar{\varepsilon}=\hbar v_{0}(|\vec{p}|+|\vec{p}+\vec{q}|)
\end{aligned}
$$


Notice also that in this regime the angle $\phi$ defined in Eq. (11) is the angle between $\vec{p}$ and the y axis. We consider the contribution to Eq. (25) from transitions between Bloch states lying within an energy region of width $\varepsilon_{\max }=\hbar v_{0} p_{\max }$ around the Fermi energy. Provided that $\varepsilon_{\max } \lesssim 2 \mathrm{eV}$, this restricts us completely to $\pi_{z}-\pi_{z}^{*}$ transitions, as graphene has no other bands in this energy region. Furthermore, the Bloch dispersion is quite accurately linear in this energy region, which corresponds to the vicinity of the Dirac $K$ points in the BZ Thus the contribution from the vicinity of one of the two inequivalent $K$ points, say $K_{1}$, is

$$
\bar{\chi}_{K S}^{\sin g: K_{1}}(\vec{q}, i u)=\frac{-1}{(2 \pi)^{2}} \int_{p<p_{\max }} \frac{2 \bar{\epsilon}}{\left.\bar{\epsilon}^{2}+(\hbar u)^{2}\right)}(1-\cos \Delta \phi) d^{2} p
$$

where $\bar{\epsilon}$ and $\phi$ are approximated as in Eq. $(28,29)$. We change from the integration variables $p_{x}, p_{y}$ to the new independent variables $P=\left(p+p^{\prime}\right) / \sqrt{2}=(|p|+|\vec{p}+\vec{q}|) / \sqrt{2}$ and $Q=$ $\left(p-p^{\prime}\right) / \sqrt{2}$ and use trigonometric identities in the triangle formed by the vectors $\vec{p}, \vec{q}$ and $\vec{p}+\vec{q}$. The integral is then elementary and gives the contribution $\bar{\chi}_{K S}^{\sin g: K_{1}}$ to the reduced response from the vicinity of just ONE of the two inequivalent Dirac points $\vec{K}_{1}, \vec{K}_{2}$. Inserting a factor two to cover contributions from the vicinity of both $\vec{K}$ points, we find $\bar{\chi}_{K S}^{\sin g}(\vec{q}, i u)=-\frac{q^{2}}{2 \hbar}\left(\frac{1}{\sqrt{v_{0}^{2} q^{2}+u^{2}}}\left[\frac{2}{\pi} \arctan \frac{w_{\max }}{\sqrt{v_{0}^{2} q^{2}+u^{2}}}\right]\right), \quad w_{\max }=\sqrt{4 p_{\max }^{2}-q^{2}} \approx 2 p_{\max }$.

Note that from Eq. (31), as the 2D wavenumber $q$ and imaginary frequency $u$ both become small, the polarizability $\alpha \equiv q^{-2} \chi_{K S}$ becomes singular, $\alpha \propto\left(v_{0}^{2} q^{2}+u^{2}\right)^{-1 / 2}$. This means that vdW theories of the Lifshitz type [13] which rely on a spatially local approximation (i.e. a $q$-independent $\alpha$ as $q \rightarrow 0$ ) are not applicable to graphene without substantial modification. This singularity also gives rise to unusual power laws $-C_{p} D^{p}$, which we will exhibit below, for the interaction energy with an object distant $D$ from the graphene plane. Note that the arctan factor in square brackets in Eq. (31) has apparently not been derived before [14], [12]. It cuts off the response at high frequencies, and restores the correct $u^{-2}$ asymptotic form in this limit. This information is required to understand dispersion (van der Waals) energies which depend on the response at all frequencies. 


\section{H Effects of finite bandwidth (non-linear $\varepsilon$ vs $q$ ), and of other bands}

The full response function $\chi_{K S}$ contains contributions from $\left(n, n^{\prime}\right)$ transitions involving bands $n$ other than the $\pi_{z}$ bands. Also we need to consider transitions to or from $\vec{k}$-points lying in the $\pi_{z}$ bands but not near the Dirac $K$ points: these were not included in $\chi_{K S}^{\sin g}$ above. These are all "gapped" transitions, however, and they lead to a finite contribution to the polarizability $a \equiv q^{-1} \chi$ as $q \rightarrow 0$. It can be shown that in this limit the response is dominated by the singular gapped contribution calculated above. As a result we can calculate the interaction of graphene with very distant $(D \rightarrow \infty)$ objects using only $\chi_{K S}^{\sin g}$. By contrast for small $D$ values near to the equilibrium geometry the dispersion force is typically doiminated by the majority "gapped" transitions.

\section{Interacting response}

The discussion above yielded the bare (Kohn-Sham) response function, in which the Coulomb interaction between the linearly responding electrons was ignored. Here we will restore these interactions within the Random Phase Approximation (RPA, Time Dependent Hartree, TDH) . There has been some discussion [14] whether graphene needs to be treated in a strongly correlated theory beyond RPA, with the conclusion that the coulomb operator is only marginally relevant. There is also evidence from the $1 \mathrm{D}$ electron gas, a definitively strongly correlated system, that the long-wavelength collective excitations, where the electrons move approximately in unison, are still treated well by the RPA. [15] Accordingly we adopt this theory here.to investigate the long-wavelength $(\vec{q} \rightarrow 0)$ response that dominates the interaction of graphene with other distant systems.

In the RPA the full response $\chi$ corresponds simply to the bare density response

$$
\chi * \delta v^{e x t}=\delta n(r, t)=\chi_{K S} *\left(\delta v^{e x t}+\delta v^{i n t}\right)
$$

due to a field that includes the internally generated TDH contributon

$$
\delta v^{\text {int }}(\vec{r}, t)=V_{\text {coul }} * \delta n
$$


(Here "*" represents spatial convolution and $V_{\text {coul }}$ is the electron-electron Coulomb interaction).

\section{J Local field effects at small $q_{\|}$}

In the present context of a system with $2 \mathrm{D}$ spatial periodicity existing in a $3 \mathrm{D}$ world with the $z$ axis perpendicular to the plane, the TDH self-consistent field equation Eq. (32) can be written

$$
\begin{aligned}
\chi_{\lambda}\left(\vec{q}, \vec{G}, \vec{G}^{\prime}, z, z^{\prime}, i u\right)= & \chi_{K S}\left(\vec{q}, \vec{G}, \vec{G}^{\prime}, z, z^{\prime}, i u\right) \\
& +\int d z^{\prime \prime} d z^{\prime \prime \prime} \sum_{\vec{G}^{\prime \prime}} \chi_{K S}\left(\vec{q}, \vec{G}, \vec{G}^{\prime \prime}, z, z^{\prime \prime}, i u\right) \\
& \times \lambda V^{\text {coul }}\left(\vec{q}+\vec{G}^{\prime \prime}, z^{\prime \prime}, z^{\prime \prime \prime}\right) \chi_{\lambda}\left(\vec{q}, \vec{G}^{\prime \prime}, \vec{G}^{\prime}, z^{\prime \prime \prime}, z^{\prime}, i u\right)
\end{aligned}
$$

where $\vec{q}$ and $\vec{G}$ are two-dimensional. $\chi_{\lambda}$ represents the response for electrons that interact with each other via a reduced Coulomb interaction $\lambda V_{\text {coul }}$, with a $\lambda$-dependent static external potential applied in order to keep the groundstate density independent of $\lambda$. This $\lambda$-dependent response is needed to implement the RPA correlation energy discussed in a later Section. The real system has $\lambda=1$.

Eq. (34) is similar to the RPA equation that leads (e.g.) to a description of plasmons in a homogeneous electron gas. Because the crystal lattice breaks translational symmetry, however, it also describes "Local Field Effects" [16]. ("LFE" used here is not to be confused with beyond-RPA correlation effects within Time Dependent Density Functional theory, which are sometimes also termed "LFE"). The LFE described here consists in the fact that a perturbing potential $\delta v$ of long wavelength $\lambda=2 \pi|\vec{q}|^{-1},|\vec{q}| \rightarrow 0$ can excite a density perturbation $\delta n$ that varies on a short spatial scale $\lambda^{\prime}=2 \pi /|q+G|$ comparable to the lattice spacing or less: this corresponds to distortion of the electron density within a unit cell. (In the present theory with only one $\pi_{z}$ Wannier function, this occurs by hopping of electrons between the two localized orbitals within the cell: there can be no distortions of 
the localized orbital density, beacuse that would require superposition with a second excited orbital.) Furthermore the short-wavelength field from this density perturbation can drive an additional response at long wavelength. In Eq. (34) these LFE couplings occur via the off-diagonal elements $\left(\vec{G} \neq \vec{G}^{\prime}\right)$ of $\chi_{K S}$.

We analyze the RPA screening equation (34) first with $\chi_{K S}$ evaluated in the onsiteapproximation. Because of the form of the bare response Eq. (24) in this approximation, we have a separation both in $\mathrm{z}$ and in $\mathrm{G}$ space:

$$
\chi_{K S}\left(\vec{q}, \vec{G}, \vec{G}^{\prime}, z, z^{\prime}, \omega=i u\right)=S_{0}(\vec{q}+\vec{G}, z) S_{0}^{*}\left(\vec{q}+\vec{G}^{\prime}, z^{\prime}\right) \bar{\chi}_{K S}(\vec{q}, i u)
$$

We will find that we can obtain a solution the same form for the interacting response:

$$
\chi_{\lambda}\left(\vec{q}, \vec{G}, \vec{G}^{\prime}, z, z^{\prime}, \omega=i u\right)=S_{0}(\vec{q}+\vec{G}, z) S_{0}^{*}\left(\vec{q}+\vec{G}^{\prime}, z^{\prime}\right) \bar{\chi}_{\lambda}(\vec{q}, i u)
$$

Putting this into the intra-layer screening equation (34) (with arguments $q$ and $\omega$ suppressed for compactness) we have

$$
\begin{aligned}
S_{0}(\vec{q}+\vec{G}, z) S_{0}^{*}\left(\vec{q}+\vec{G}^{\prime}, z^{\prime}\right) \bar{\chi}_{\lambda}= & S_{0}(\vec{q}+\vec{G}, z) S_{0}^{*}\left(\vec{q}+\vec{G}^{\prime}, z^{\prime}\right) \bar{\chi}_{K S}+\bar{\chi}_{K S}(\vec{q}, i u) S_{0}(\vec{q}+\vec{G}, z) \\
& \times \sum_{\vec{G}^{\prime \prime}}\left(\int S_{0}^{*}\left(\vec{q}+\vec{G}^{\prime \prime}, z^{\prime \prime}\right) V^{c o u l}\left(\vec{q}+\vec{G}^{\prime \prime}, z^{\prime \prime}, z^{\prime \prime \prime}\right) S_{0}\left(\vec{q}+\vec{G}^{\prime \prime}, z^{\prime \prime \prime}\right) d z^{\prime \prime} d z^{\prime \prime \prime}\right) \\
& \times S_{0}^{*}\left(\vec{q}+\vec{G}^{\prime}, z^{\prime}\right) \bar{\chi}_{\lambda}(\vec{q}, i u)
\end{aligned}
$$

Here

$$
V^{\text {coul }}\left(\vec{k}, z, z^{\prime}\right)=\frac{2 \pi e^{2}}{|\vec{k}|} \exp \left(-|\vec{k}|\left|z-z^{\prime}\right|\right), \quad \vec{k} \text { is } 2 D
$$

is the 2D fourier transform of the electron-electron Coulomb interaction. Since every term in Eq. (36) depends on $\left(z, z^{\prime}\right)$ and $\left(\vec{G}, \vec{G}^{\prime}\right)$ through the same factor $S_{0}(\vec{q}+\vec{G}, z) S_{0}^{*}\left(\vec{q}+\vec{G}^{\prime}, z^{\prime}\right)$, the Ansatz (35) is confirmed to give an exact solution of the screening equation, with

$$
\bar{\chi}_{\lambda}=\bar{\chi}_{0}+\bar{\chi}_{0} W \bar{\chi}_{\lambda}, \therefore \quad \bar{\chi}_{\lambda}(\vec{q}, i u)=\frac{\bar{\chi}_{0}(\vec{q}, i u)}{1-\bar{\chi}_{0}(\vec{q}, i u) W(\vec{q}, i u)} .
$$

Here

$$
W(\vec{q}, i u)=\sum_{\vec{G}^{\prime \prime}} \iint S_{0}^{*}\left(\vec{q}+\vec{G}^{\prime \prime}, z^{\prime \prime}\right) V^{\text {coul }}\left(\vec{q}+\vec{G}^{\prime \prime}, z^{\prime \prime}, z^{\prime \prime \prime}\right) S_{0}\left(\vec{q}+\vec{G}^{\prime \prime}, z^{\prime \prime \prime}\right) d z^{\prime \prime} d z^{\prime \prime \prime}
$$


is the coulomb potential smeared over z and z', and summed over reciprocal lattice vectors.

Note that because of the dominance of the $\vec{G}^{\prime \prime}=\overrightarrow{0}$ term in Eq. (38), for $q \rightarrow 0$ we can write

$$
\begin{aligned}
W(\vec{q}, i u) & \sim\left(\int S_{0}^{*}\left(\vec{q}, z^{\prime \prime}\right) d z^{\prime \prime}\right) \frac{2 \pi e^{2}}{q}\left(\int S_{0}\left(\vec{q}, z^{\prime \prime \prime}\right) d z^{\prime \prime \prime}\right) \\
& \sim 1 \frac{2 \pi e^{2}}{q} 1=\frac{2 \pi e^{2}}{q} \text { as } q \rightarrow 0
\end{aligned}
$$

This means that for $q \rightarrow 0$, the screened response Eq. (37) can be calculated from $\chi_{0}(\vec{G}=$ $\left.\overrightarrow{0}, \vec{G}^{\prime}=\overrightarrow{0}\right)$ alone, ignoring the existence of other $\left(\vec{G}, \vec{G}^{\prime}\right)$ components of $\chi_{0}$ and $\chi_{\lambda}$.

Note that the above demonstration would be irrelevant for a highly insulating crystal, where with a single Wannier function on each site as used here, charge cannot be moved by an external potential, when only one-site transitions are allowed. Thus the on-site approximation would give zero response whereas it certainly does not do so here as evidenced by Eq (31). Here, the degeneracy of the problem means that applying a potential to one site does affect the others, via the degenerate overlap effects already included when deriving the mixing coeficients $e$ (see Eq. (11)).

The effects of the near-neighbor terms in these local-field considerations can be solved in principle by inverting an $8 \times 8$ matrix [11] but the details have not yet been carried through. For the moment we will assume, as have previous authors [17],[12]], that the local field effects are unimportant as $q_{\|} \rightarrow 0$, so that we only need to look at the z-integrated, $\vec{G}=\vec{G}^{\prime}=\overrightarrow{0}$ component of the response, which from Eq. (37) and Eq. (26) is

$$
\begin{aligned}
\bar{\chi}(\vec{q}, i u) & =\frac{\bar{\chi}_{0}(\vec{q}, i u)}{1-\frac{2 \pi e^{2}}{q} \bar{\chi}_{0}(\vec{q}, i u)} \\
& =-\frac{q}{2 \hbar v_{0}} \frac{A}{\left(1+\frac{u^{2}}{v_{0}^{2} q^{2}}\right)^{1 / 2}+\frac{\pi e^{2}}{\hbar v_{0}} A}, \quad A=\frac{2}{\pi} \arctan \frac{w_{\max }}{\sqrt{v_{0}^{2} q^{2}+u^{2}}} .
\end{aligned}
$$

Eq. (39) represents the contribution from gapless transitions, and is dominant for small $q$ and $u$ values. 


\section{INTERACTION ENERGY OF OF GRAPHENE WITH DISTANT OBJECTS}

\section{A Electrostatic interaction}

The graphene electronic groundstate density $n_{0}(\vec{r})$ does interact directly with the external charge distribution, but a graphene sheet located at $z=0$ has a density $n_{0}(\vec{r}) \approx$ $\delta(z) \sum_{\vec{G} \neq 0} n_{0 \vec{G}} \exp (i \vec{G} \cdot r)$ where $\vec{G}$ is a $2 \mathrm{D}$ reciprocal lattice vector and the spatially uniform component $n_{0 \vec{G}=\overrightarrow{0}}$ is zero for an electrically neutral graphene sheet. This density creates an electrostatic potential, $V_{0}(D)$ at a point distant $D$ from the graphene plane, equal to

$$
V_{0}(D)=\sum_{\vec{G} \neq 0} n_{0 \vec{G}} \exp (i \vec{G} \cdot r) \frac{2 \pi e^{2}}{G} e^{-G D}
$$

Since $G \equiv|G|=O(2 n \pi / a), V_{0}(D)$ is exponentially damped when $D>a$ where $a$ is the graphene lattice spacing. Thus the direct electrostatic interaction can be ignored for distant objects.

\section{B Induction interaction of graphene with a distant fixed localized external charge distribution}

At large distances $D>>a$, the dominant interaction with an external fixed charge distribution is therefore due to induction, whereby the field of the fixed external charges distorts the electronic charge distribution in the graphene sheet, resulting in both a change in electrostatic energy and a change in the electronic zero-point kinetic energy. Both of these contributions are included when one calculates the work done by an external agent to switch on the external potential adiabatically. The work to increase the external potential from $\lambda v^{e x t}$ to $(\lambda+d \lambda) v^{e x t}$ is, to 1st order in $d \lambda$,

$$
d E=d\left(\left\langle\Psi_{\lambda}|\hat{H}|_{\lambda} \Psi_{\lambda}\right\rangle\right)=d\left(\left\langle\Psi_{\lambda}\left|\hat{T}+\vec{V}^{e e}+\lambda \hat{V}^{e x t}\right| \Psi_{\lambda}\right\rangle\right)=\left\langle\Psi_{\lambda}\left|d \lambda \hat{V}^{e x t}\right| \Psi_{\lambda}\right\rangle
$$

where the last equality follows since $\left\langle\Psi_{\lambda}\left|\hat{H}_{\lambda=\lambda_{0}}\right| \Psi_{\lambda}\right\rangle$ is stationary for small changes in $\lambda$ about the point $\lambda_{0}$ (Feynman-Hellmann theorem). The perturbation $n_{1}$ to the graphene 
electronic density has long-wavelength components that can couple to the external charge. Then

$$
\begin{aligned}
d E & =\left\langle\Psi_{\lambda}\left|d \lambda \hat{V}^{e x t}\right| \Psi_{\lambda}\right\rangle=d \lambda \int v^{e x t}(\vec{r}) n_{1}(\vec{r}) d \vec{r} \\
& =d \lambda \int v^{e x t}(\vec{r}) \int \chi\left(\vec{r}, \vec{r}^{\prime}, \omega=0\right) \lambda v^{e x t}\left(\vec{r}^{\prime}\right) d \vec{r}^{\prime} d \vec{r}+O\left(\lambda^{2}\right)
\end{aligned}
$$

The induction energy to second order in the external perturbation is then

$$
\begin{aligned}
E^{(2) \text { induction }} & =\int_{0}^{1} d E=\left(\int_{0}^{1} \lambda d \lambda\right) \iint v^{e x t}(\vec{r}) \chi\left(\vec{r}, \vec{r}^{\prime}, \omega=0\right) v^{e x t}\left(\vec{r}^{\prime}\right) d \vec{r}^{\prime} d \vec{r} \\
& =\frac{1}{2} \iint v^{e x t}(\vec{r}) \chi\left(\vec{r}, \vec{r}^{\prime}, \omega=0\right) v^{e x t}\left(\vec{r}^{\prime}\right) d \vec{r}^{\prime} d \vec{r} \\
& =\frac{1}{2} \frac{1}{(2 \pi)^{2}} \int d \overrightarrow{q_{\|}} \bar{\chi}\left(\vec{q}_{\|}, \omega=0\right) v^{e x t}\left(\vec{q}_{\|}, z=0\right) v^{e x t}\left(-\vec{q}_{\|}, z^{\prime}=0\right)
\end{aligned}
$$

where $\bar{\chi}$ is the $z$-integrated graphene electronic response.introduced earlier.

\section{Point charge near graphene}

A point charge $Q$ at position $(0,0, D)$ creates a potential energy

$$
v^{e x t}\left(q_{\|}, z=0\right)=-\frac{2 \pi Q e}{q_{\|}} e^{-q_{\|} D}
$$

and Eq. (42) becomes

$$
E^{i n d, Q}=\frac{1}{2} \frac{(2 \pi Q e)^{2} 1}{(2 \pi)^{2}} \int d \vec{q}_{\|} q_{\|}^{-2} e^{-2 q_{\| \mid} D} \bar{\chi}\left(\vec{q}_{\|}, \omega=0\right)
$$

For graphene, from Eq. (39),

$$
\bar{\chi}\left(\vec{q}_{\|}, i u\right)=-\frac{q_{\|}}{2 \hbar v_{0}}\left(\frac{\frac{2}{\pi} \arctan \frac{w_{\max }}{v_{0} q_{\|}}}{\sqrt{1+\left(u / v_{0} q_{\|}\right)^{2}}+\frac{\pi e^{2}}{\hbar v_{0}} \frac{2}{\pi} \arctan \frac{w_{\max }}{v_{0} q_{\|}}}\right)+\Delta \chi
$$

where $\Delta \chi$ represents the nonsingular "gapped" contribution from $\pi_{z}-\pi_{z}^{*}$ transitions between points $\vec{k}$ far from the Dirac points, as well as from gapped transitons involving other Bloch bands. Note that $\Delta \chi \rightarrow \alpha q_{\|}^{2}$ as $q_{\|} \rightarrow 0$ where $\alpha$ remains finite as $q_{\|} \rightarrow 0$.Then provided 
that the only $q_{\|}$values that are sampled satisfy $q_{\|}<2 \pi v_{0} \hbar \alpha$ (so that $\Delta \chi$ is negligible) and $q_{\|}<w_{\max } / v_{0}$ (so that $\frac{2}{\pi} \arctan \frac{w_{\max }}{v_{0} q_{\|}} \approx 1$ ) we can approximate

$$
\bar{\chi}(\vec{q} \|, \omega=0)=-\frac{q_{\|}}{2 v_{0} \hbar} \frac{1}{1+\frac{\pi e^{2}}{\hbar v_{0}}}
$$

In the examples below, because of the factor $\exp \left(-q_{\|} D\right)$, the simplified low- $q_{\|}$form Eq. (44) is valid provided that the interacting object is distant by a separation $D$ satisfying

$$
D>>2 \pi v_{0} \hbar \alpha, D>>\frac{v_{0}}{w_{\max }} .
$$

Then the induction energy is

$$
E^{\text {ind,Q-graphene }}=-\frac{(Q e)^{2} 2 \pi}{2(2 \hbar) v_{0}} \int_{0}^{\infty} e^{-2 q_{\|} D} \frac{1}{1+\frac{\pi e^{2}}{\hbar v_{0}}} d q_{\|}=-\frac{Q^{2}}{4 D} \frac{\frac{\pi e^{2}}{\hbar v_{0}}}{1+\frac{\pi e^{2}}{\hbar v_{0}}}
$$

Since $\pi e^{2} /\left(\hbar v_{0}\right) \approx 7$, this result represents a near-perfect image charge behavior although graphene is only one atom thick, and this is clearly due to the semi-metallic nature of graphene. For comparison, a 2D electron gas (2DEG) with areal electron number density $n_{0}$ has $\chi=-n_{0} q_{\|}^{2} m^{-1} /\left(\omega^{2}-\omega_{P 2 D}^{2}\right)$ where the 2 D plasma frequency is $\omega_{P 2 D}=2 \pi n_{0} e^{2} q_{\|} / m$. The static response is then

$$
\bar{\chi}^{2 D E G}\left(q_{\|}, \omega=0\right)=-q_{\|} /\left(2 \pi e^{2}\right)
$$

and then Eq. (43) gives

$$
E^{i n d, Q-2 D E G}=-\frac{Q^{2}}{4 D}
$$

which is perfect image behavior, the same as for an infinitely thick metal slab.

For a 2D insulator (with in-plane polarizability $a_{0}$ only, for simplicity) we have

$$
\chi^{2 D I n s}=-q_{\|}^{2} \alpha_{0}
$$

for small $q_{\|}$and Eq. (43) gives

$$
E^{i n d, Q-2 D I n s}=-\pi \alpha_{0} \frac{Q^{2} e^{2}}{4 D^{2}}
$$

More detailed calculations for external point charges, including effects of finite velocity, can be found in Ref [55]. However the simple closed result (46) above does not appear to have been given explicitly in [55]. 


\section{Fixed dipole}

For simplicity we consider a dipole with moment $P$ located at $(0,0, D)$ and oriented parallel to the graphene plane. (The case of more general orientation is given elsewhere [18]). The electron potential energy function at the graphene plane $z=0$, due to such a dipole, is

$$
v^{e x t}\left(\vec{q}_{\|}, z=0\right)=\left[-i P q_{x}\right] \frac{2 \pi e}{q_{\|}} e^{-q_{||} D} .
$$

Putting Eq. (51) into Eq. (42) and using Eq. (44) we find the following result provided $Z$ is sufficiently large that the conditions Eq. (45) are met :

$$
E^{\text {ind,dipole-graphene }}=-D^{-3} \frac{P^{2}}{16} \frac{\frac{e^{2}}{\hbar v_{0}}}{1+\frac{e^{2}}{\hbar v_{0}}}
$$

The result for a dipole interacting with a 2DEG is found from Eq. (47), Eq. (51) and Eq. (42):

$$
E^{\text {ind,dipole-2DEG }}=-D^{-3} \frac{P^{2}}{16}
$$

For a 2D insulator in place of graphene we find from Eq. (49), Eq. (51) and Eq. (42)

$$
E^{\text {Ind,dipole-2DInsulator }} \approx-D^{-4} \frac{3 \pi e^{2} \alpha_{0} P^{2}}{16}
$$

Note that the graphene results Eq. $(46,52)$ are valid only at very large separations $D$, often $5 \mathrm{~nm}$ or more. At more modest separations we cannot ignore the induction energy from the non- $\pi_{z}-\pi_{z}^{*}$ transitions. Furthermore at these separations we have to consider crossscreening within the graphene plane between the semi-metallic response from the gapless $\pi_{z}-$ $\pi_{z}^{*}$ transitions and the semiconductor-like response from the remaining gapped transitions. At still smaller separations, electron overlap effects such as exchange come into play, and furthermore the second order perturbative approach could also break down. Local-field effects in the screening will also come into play as the interaction is no longer restricted to small wavevectors $q_{\|}$In this regime LDA/GGA calculations (see below) would be a method of choice for the induction physics. 


\section{E Dispersion interaction of graphene with distant objects}

The induction energy discussed in the last Section consisted of (primarily) single-electron groundstate kinetic energy, as well as electrostatic energy, in the groundstate of a compound system of graphene and an external object. Hence the induction energy should be reproducible in a sufficiently accurate Electron Density Functional Theory calculation using common approximations such as the Local Density Approximation (LDA) or Generalized Gradient Approximation (GGA). Graphene also interacts with other objects via dispersion (van der Waals, vdW) forces. For widely spaced objects, the latter are NOT describable at all within the LDA or GGA because the electronic correlations involved are very longranged, negating the assumptions of the semi-local theories. [19],[20]. The long ranged vdW correlations are, however captured (at least qualitatively and often quantitatively) by theories in the Random Phase Approximation (RPA) class. One way to obtain such theories is to use the Adiabatic Connection Fluctuation Dissipation (ACFD) approach (see e.g. $[21],[22])$ Here one calculates the electronic density response $\chi_{\lambda}$ of the coupled system with a modified electron-electron interaction $\lambda e^{2} /\left|\vec{r}-\vec{r}^{\prime}\right|$, then uses this $\chi_{\lambda}$ in the Fluctuation Dissipation Theorem [23] to calculate the groundstate electron pair distribution $n_{2 \lambda}\left(\vec{r}, \vec{r}^{\prime}\right)$. This is usually done in such a way that the groundstate electron density and hence the bare response $\chi_{K S}$ are independent of $\lambda$. Then one applies the Adiabatic Connection formula $[21],[22],[24]$ to obtain the groundstate energy of the fully interacting $(\lambda=1)$ system as the integral of a coulomb energy over the coupling strength $\dot{\lambda}$ The exact ACFD theorem is

$$
E_{c}=-\frac{\hbar}{2 \pi} \int_{0}^{1} d \lambda \int d \vec{r} d \vec{r}^{\prime} \frac{e^{2}}{\left|\vec{r}-\vec{r}^{\prime}\right|} \int_{0}^{\infty}\left(\chi_{\lambda}\left(\vec{r}, \vec{r}^{\prime}, i u\right)-\chi_{\lambda=0}\left(\vec{r}, \vec{r}^{\prime}, i u\right)\right) d u
$$

where $\chi_{\lambda=0} \equiv \chi_{K S}$ (see e.g. [25]). Here we will use Eq. (55) to study the vdW interaction between two graphene planes, filling in some of the details from [3]. We need the response of the two parallel interacting sheets separated by distance $D$. The response of each sheet, with all intra-sheet interactions included, will be taken from the $z, z$ Integrated response Eq. (39). For large $D$ where electronic overlap is negligible, the distribution of $\pi_{z}$ charge 
across the thickness of the sheet is immaterial to the inter-sheet interaction, so we assume a delta functon

$$
\begin{aligned}
\chi_{\lambda}\left(\vec{r}, \vec{r}^{\prime} i u ; D\right)= & \int d^{2} q_{\|} e^{i q_{\|} \cdot\left(\vec{r}-r^{\prime}\right)}\left[\left(\delta(z) \delta\left(z^{\prime}\right)+\delta(z-D) \delta\left(z^{\prime}-D\right)\right) \chi_{11 \lambda}\left(q_{\|}, i u, D\right)\right. \\
& \left.+\left(\delta(z) \delta\left(z^{\prime}-D\right)+\delta\left(z^{\prime}\right) \delta(z-D)\right) \chi_{12 \lambda}\left(q_{\|}, i u, D\right)\right]
\end{aligned}
$$

The response $\chi_{\lambda}\left(\vec{r}, \vec{r}^{\prime} i u ; D \rightarrow \infty\right)$ in the absence of inter-sheet interactions is of the same form with

$$
\chi_{11 \lambda}\left(q_{\|}, i u, D \rightarrow \infty\right)=\bar{\chi}_{\lambda}\left(q_{\|}, i u\right), \quad \chi_{12 \lambda}\left(q_{\|}, i u, D \rightarrow \infty\right)=0
$$

Here $\bar{\chi}_{\lambda}$ is given by Eq. (39) and includes the interactions amongst the electrons of a single sheet at RPA level. The RPA self-consistent field equations (see Eq. $(32,33)$ )for the timedependent density perturbations $n_{1}\left(q_{\|}, i u\right)$ and $n_{2}\left(q_{\|}, i u\right)$ on the two coupled graphene sheets are

$$
\begin{aligned}
& n_{1}=\bar{\chi}_{\lambda}\left(q_{\|}, i u\right)\left(v_{1}^{e x t}+\lambda V_{12}\left(q_{\|}, D\right) n_{2}\right) \\
& n_{2}=\bar{\chi}_{\lambda}\left(q_{\|}, i u\right)\left(\lambda V_{12}\left(q_{\|}, D\right) n_{1}+v_{2}^{e x t}\right)
\end{aligned}
$$

where $V_{12}\left(q_{\|}, D\right)=2 \pi e^{2} q_{\|}^{-1} e^{-q_{\|} D}$ is the 2 D Fourier transform of the Coulomb potential. By solving Eq. (57) for the case $v_{1} \neq 0, v_{2}=0$ we obtain

$$
\begin{aligned}
& \chi_{11 \lambda}\left(q_{\|}, i u ; D\right)=n_{1} / v_{1}=\bar{\chi}_{\lambda} /\left(1-\left(\lambda \bar{\chi}_{\lambda} V_{12}\right)^{2}\right) \\
& \chi_{12 \lambda}\left(q_{\|}, i u ; D\right)=\bar{\chi}_{\lambda} \lambda V_{12} \chi_{11 \lambda}\left(q_{\|}, i u ; D\right)
\end{aligned}
$$

(we note a typographical error in Ref [3] where the "square" on the last term in Eq. (58) was omitted). We now put Eq. (56), Eq. (58) and Eq. (59) into Eq. (55). Noting that $\chi_{K S}=\chi_{\lambda=0}$ is independent of $D$, we find that the $D$-dependent part of the correlation energy (the "cross-correlation" energy) gives the vdW interaction energy per unit area:

$$
\frac{E^{v d W}}{A}=\frac{E_{c}(D)-E_{c}(D \rightarrow \infty)}{A}=-\frac{\hbar}{(2 \pi)} \int_{0}^{1} d \lambda \int_{0}^{\infty} d u \int \frac{d^{2} q_{\|}}{(2 \pi)^{2}}\left(\lambda V_{11} \Delta \chi_{11 \lambda}+\lambda V_{12} \chi_{12 \lambda}\right)
$$

Here $\Delta \chi_{11 \lambda}=\chi_{11 \lambda}\left(q_{\|}, i u ; D\right)-\chi_{11 \lambda}\left(q_{\|}, i u ; \infty\right)$. Both $\Delta \chi_{11 \lambda}$ and $V_{12} \chi_{12 \lambda}$ are exponentially small, $\propto \exp \left(-2 q_{||} D\right)$ for large $D$, so that the integral Eq. (60) is restricted to small values 
of $q_{\|}$. For sufficiently large $D$ and small $u$ values this means that the arctan cutoff factor in Eq. (31) equals 1. At larger $u$, the integral Eq. (60) turns out to be convergent without the cutoff, so the cutoff factor is not significantly called into play. Thus instead of Eq. (31) we can take

$$
\begin{aligned}
\bar{\chi}_{K S}\left(\vec{q}_{\|}, i u\right) & =-\frac{q_{\|}^{2}}{2 \hbar} \frac{1}{\sqrt{v_{0}^{2} q_{\|}^{2}+u^{2}}}, \\
\bar{\chi}_{\lambda} & =\frac{\bar{\chi}_{K S}}{1-\lambda \frac{2 \pi e^{2}}{q_{\|}} \bar{\chi}_{K S}}=-\frac{q_{\|}}{2 \hbar v_{0}} \frac{1}{\sqrt{1+\left(u / v_{0} q_{\|}\right)^{2}}+\lambda \frac{\pi e^{2}}{\hbar v_{0}}}
\end{aligned}
$$

everywhere in Eq. (60). Following back through Eqs. (61), (58), (59), we find that all occurrences of $\bar{\chi}_{K S}$ in Eq. (60) come multiplied by the $2 \mathrm{D}$ coulomb interaction $\propto q_{\|}^{-1}$ and so have form $V \bar{\chi}_{K S} \propto\left(1+\left(u / v_{0} q_{\|}\right)^{2}\right)^{-1 / 2} \operatorname{or}\left(1+\left(u / v_{0} q_{\|}\right)^{2}\right)^{-1 / 2} \exp \left(-q_{||} D\right)$. Thus the only occurrence of $u$ is via the dimensionless variable $U=u /\left(v_{0} q_{\|}\right)$and the remaining dependence on $q_{\|}$is via the dimensionless variable $Q \equiv q_{\|} D$. Therefore Eq. (60) is of the form

$$
\begin{aligned}
E^{v d W} / A & =\hbar \int d q_{\|} q_{\|} \int_{0}^{\infty}\left(v_{0} q_{\|} d U\right) \int d \lambda F_{\lambda}\left(D q_{\|}, U\right) \\
& =D^{-3} \hbar v_{0} \int_{0}^{\infty} d Q Q^{2} \int_{0}^{\infty} d U \int_{0}^{1} d \lambda F_{\lambda}(Q, U)
\end{aligned}
$$

which is proportional to $D^{-3}$. By contrast all functionals (pair summation, vdWDF) that depend on pairwise additivity of $R^{-6} \mathrm{vdW}$ energies between pairs of atoms (or of elements) predict $E \propto D^{-4}$. Numerical evaluation of the integral Eq. (62) with graphene parameters gives [3]

$$
E^{v d W} / A=-7.75 \times 10^{-2} \hbar v_{0} D^{-3}
$$

This result arises because of the unusual behavior of the bare graphene response, $\bar{\chi}_{K S} \propto$ $q_{\|}\left(1+\left(u / v_{0} q_{\|}\right)^{2}\right)^{-1 / 2}$ as $q_{\|}$and $u$ go to zero. By contrast, a metal has $\bar{\chi}_{K S} \propto q_{\|}^{2} / u^{2}$ and the analysis above can be repeated for a $2 \mathrm{D}$ metal to give $E^{v d W} / A \propto D^{-5 / 2}$. This result has been known for some time [26],[27]. For a 2D insulator, $\bar{\chi}_{K S} \propto q^{2} / \omega_{0}^{2}$ and the above analysis gives the conventional result $E^{v d W} / A \propto D^{-4}$. as predicted for $2 \mathrm{D}$ geometry by all pairwiseadditive theories Thus both the response function and the vdW power law of graphene are 
intermediate betwen 2D metal and 2D insulator behavior. This is not surprising since the graphene Bloch bandstructure is gapless but has a zero density of states where the $\pi_{z}$ and $\pi_{z}^{*}$ bands touch: this is again intermediate between a metal with no gap but a finite DOS, and an insulator with a finite gap and a zero DOS at the bandedge.

\section{F Dispersion interaction between graphene and a distant polarizable molecule}

The above RPA analysis can be repeated to obtain the vdW interaction for the case of a graphene sheet and a distant point polarizable dipole, representing e.g. a hydrogen molecule, which has no permanent dipole. The details will be given elsewhere [18], but the result is that an insulating sheet gives $E^{v d W} \propto-D^{-4}$, a $2 \mathrm{D}$ metal gives $E^{v d W} \propto-D^{-3}$, and graphene gives again an intermediate result $E^{v d W} \propto-D^{-4} \ln \left(D / D_{0}\right)$.

\section{G Limitations of the asymptotic theories}

The above analytic results rely on the small- $q_{||}$approximation and are valid only for very large separations $D>>v_{0} / w_{\max }, D>>2 \pi v_{0} \hbar \alpha$-see Eq. (45). This limits the analysis in practice to separations of $O(5 \mathrm{~nm})$ or more. Furthermore the role of the Local Field Effects on a graphene sheet is still uncertain if effects beyond the on-site approxination for $\chi_{K S}$ are important, and this could lead to a modification of the numerical front factor in formulae such as Eq. (63).

For finite temperatures a low-density electron gas will be created in graphene, presumably resulting in a 2DEG-like $D^{-5 / 2}$ tail in the graphene-graphene interaction for even larger $D$, outside the $D^{-3}$ region. It is quite possible, however, that this will be suppressed by electromagnetic retardation of the vdW interaction, neglected in the present theory [27]. Similar considerations apply to doped graphene, which resembles a 2D metal in some respects. 


\section{INTERACTIONS OF GRAPHENE WITH CLOSER OBJECTS}

For an accurate analysis of graphene interactions with other objects at smaller separations down to contact, knowledge of the low- $\vec{q}$ properties of the graphene response is no longer sufficient. The primary difficulty in dealing with this regime is that dispersion forces are important, and these are not dealt with correctly by common semi-local models of electron interactions, based on the LDA or GGA.

\section{A LDA for graphitics}

It is therefore perhaps surprising that LDA calculations predict the lattice spacing of graphite in very good agreement with experiment. (See e.g. [4].) However, the LDA layer binding energy of graphite is now known to be considerably too small, a fact that was obscured for years because of the relatively poor precision and large spread of the few rather indirectly-obtained experimental numbers [28],[29],[30]. Furthermore, accurate and reasonably consistent experimental determinations have been made of the $C_{33}$ (interlayer stretching) elastic constant of graphite, and LDA does not reproduce this well [4]. This situation is unsatisfactory because quite small energies can be crucial in distinguishing between structures (e.g. AB graphite vs ABC graphite).

\section{B Theories with explicit dispersion physics.}

There have been many calculations of the interaction of graphene or graphite with various objects using thories that contain explicit vdW corrections to LDA/GGA [31],[32],[33],[34]. There have also been fully empirical calculations using van der Waals pair potentials of Lennard-Jones type (e.g. [35]). A possible difficulty here is that virtually all these calculations embodied the pair-additive "sum of $R^{-6}$ " physics, either by explicit assumption [35] or by virtue of approximations made in the coulomb screening physics when deriving the theory [36],[31],[32],[33],[34]. In addition to giving inappropriate asymptotics of the distant 
interaction as discussed above (which may not be relevant to the close binding physics), the pairwise additive approach has recently been found to fail significantly for anisotropic polarizable objects even at smaller separations [37],[38],[39] . Therefore it is not clear how reliable pair-additive theories are for the delicate energetics of cohesion of graphene layers. Direct experiments are quite difficult, so the best hope of a definitive answer in the near future would seem to lie in an adequate many-electron theory, including electron correlation terms that reproduce the very nonlocal coupling between distant electron charge fluctuations that are the essence of the dispersion interaction (see e.g. [20],[19]).

Two such classes of theory are now becoming feasible for practical nonlocal electron correlation energy calculations on large periodic systems: the RPA and related theories, and the Diffusion Monte Carlo approach.

\section{RPA correlation energy for graphitics at arbitrary separation}

The RPA correlation energy was introduced above via the "ACFD" approach. In that context it can be thought of as generating groundstate correlations using information from the time-varying internal fields in a Time Dependent Hartree approximation to the electronic response. From this point of view the RPA can be generalized by extending from TDH to Time Dependent Density Functional Theory response [40]. Equally, the ("direct") RPA can be generated from systematic all-order perturbation theory (e.g. Feynman diagrams [41] or Coupled-Cluster theory [42]) via a sum of rings of open bubbles. Generalizations can be obtained by including ladder diagrams in various ways e.g. [43] and by "range separation" e.g. [44].

The simplest version, the "direct" RPA (without ladders) considered here, is known to over-estimate the short-ranged part of the electronic correlation hole quite severely, and also suffers from orbital self interaction. Because of the former effect it often gives poor total correlation energies, but it is much better for energy differences betwen states of equal electron number [45]. Because of the self-interaction it often gives a poor description of 
bond stretching in small molecules, where highly localized orbitals become less so [46],[47]. However the valence orbitals involved in graphitic interactions, particularly the $\pi_{z}$ orbitals, are quite spatially delocalized and self-interaction is therefore irrelevant to the physics of graphitic cohesion, making these problems suitable for RPA treatment. Indeed the RPA has been shown [48] to be essentially correct for the long-ranged correlation physics inherent in dispersion forces, and this includes non-pairwise-additive effects that are missed in most current numerical approaches to large systems.

Over the past decade it has become practical [49],[50], [51] to compute exact-exchange plus RPA correlation energy for crystalline solids, using the realistic Bloch bandstructure from an existing DFT solid state code (e.g. ABINIT or VASP). This data is then inserted into Eq. (16) and used in the ACFD or an equivalent method to obtain the electronic correlation energy. Harl and Kresse [51] recently obtained very good results for several properties of a wide variety of crystals this way, including some weakly bonded "vdW" crystals. These can be very large calculations. Despite this general progress, graphitic systems remain very difficult because of the need to perform the Brillouin zone integration over $\vec{p}$ in Eq. (16) using a very fine mesh to capture the conical gapless behavior of the Bloch bands at the Dirac points. This is demanding for the correlation energy calculation and even more so for the exact exchange energy calculation. Indeed a sufficiently converged result ( $48 \pm 1 \mathrm{meV}$ atom) for the layer binding energy of graphite has only very recently been obtained, along with the binding energy curve out to a layer separation round $1.5 \mathrm{~nm}$.[4]. Good agreement with experiment was obtained both for the equilibrium layer spacing and the $C_{33}$ elastic constant, and the $48 \mathrm{meV}$ binding energy figure is a new benchmark (see also the DMC discussion below) because of the large uncertainties in the sparse experimental data.. By considering transitions only within a restricted range of energies it was also possible [4] to sample the graphite BZ even more finely and so obtain reliable correlation energy results out to a layer separation $D=3 \mathrm{~nm}$. Via this approach an $E=-c D^{-3}$ power law was obtained for the first time in a realistic numerical calculation, essentially in agreement with Eq. (62), or more precisely with its generalization to a many-layered systems [52],[53]. At these values 
of $D$, the $D^{-3}$ contribution is still quite small relative to the total correlation energy from all transitions, however.

Presently, no results for stretched graphite are available from the various higher manybody theories that correct the short-ranged diseases of the direct RPA. However, it is known that, in a jellium slab analog of bigraphene, TDDFT corrections to the short range correlations in RPA made essentially no difference to the layer binding energy [54]. This accords with the reasoning given above for the validity of the RPA in these $\pi$-bonded systems.

\section{Diffusion Monte Carlo calculation of stretched graphite}

It is now also possible to obtain electronic correlation energies of solids via Diffusion Monte Carlo techniques, i.e essentially by stochastic sampling of accurate numerical solutions of the many-electron Schrodinger equation for a finite number of $N$ of electrons[55] . Like the RPA and its relatives, this method can also produce the long-ranged correlations that create the dispersion interaction. Apart from statistical noise, and the fixed-node approximation (which is probably not a problem for graphitics), DMC if converged should give the exact result. The main limiting feature of DMC in the present context is the need for a finite repeat cell to mimic an infinite solid using only a finite number of electrons. In the vdW context this could be problematic since the completely correlated motions of an electron and its distant "ghost" in the next cell will mimic the correlations inherent in the van der Waals interaction. That is, the periodic finite-cell approach can introduce spurious dispersion-like correlation energy contributions.

Recently the DMC was used [55] to compute the layer binding energy curve $E(D)$ for stretched graphite. The binding energy was $56 \pm 5 \mathrm{meV} /$ atom, though this error estimate probably did not account for the finite-size "vdW effect" described above, relative to cell size in the layer-parallel directions, since the size extrapolation was based on LDA physics. It is not clear, therefore, whether the small difference in binding energy between the DMC result and the $48 m e V /$ atom prediction from the RPA [4] is due to short-ranged physics omitted 
by the RPA, or simply to cell size convergence issues in DMC. Experiments done to date are of little help here, as reasonably credible work has given binding numbers ranging from 35 to $62 \mathrm{meV} /$ atom. The RPA results were well converged with respect to $k$ point spacing, roughly equivalent to cell size convergence in the DMC approach.

\section{E High-level calculations of molecules physisorbed on graphene}

Energy calculations for molecules absorbed on graphene can be performed easily within LDA, and reasonably easily within pair additive dispersion corrected theories [32],[33]. The problem once more is the uncertainty about non-additive vdW effects due to the highly polarizable semi-metallic nature of graphene. To elucidate this, several groups are currently workng on this problem using full RPA and/or DMC correlation energy methods. No definitive results are yet available, to this author's knowledge.

\section{FUTURE DIRECTIONS}

Concerning the analytic treatment of the asymptotic interactions of graphene with distant objects, more work needs to be done to elucidate the role of local field effects in the Coulomb screening within a graphene layer, as discussed above.

It should be possible to include simultaneously and analytically the unusual $\pi_{z}$ contribution to the graphene response, along with a more conventional semiconducting contributon from the gapped electronic transitions involving other bands. This should allow analytic work to treat smaller separations though still in the regime of negligible electron cloud overlap.

Concerning realistic prediction of dispersion energetics of graphene in close contact with other systens, the RPA and DMC calculations for stretched graphite were about at the limit of feasibilty with current software and technology, and graphitic systems in more realistic technologically interesting geometries cannot presently be modelled by these means. 
Perhaps a combination of numerical plane-wave-code RPA, or DMC, calculations on a coarse $\vec{k}$ grid (RPA), or small repeat cell (DMC), can be used along with a semi-analytic correction using the tight binding-based theories summarized in earlier sections the present work.

\section{SUMMARY}

It has been pointed out that graphene has unusual properties not only in the area of electronic transport, Hall effect etc, but also with regard to its interaction energy with other nano- and micro-structures. Like the better-known transport properties, these cohesive properties are anomalous because of the gapless semi-metallic nature of electronic excitations in graphene. Many of the results reported here are published or in press, but the analyses of local field effects and finite bandwidth effects in the electronic response of graphene are new.

In the asymptotic regime of large separations, the induction energy of a localized charge distribution distant $D$ from graphene can be calculated using the static linear desity response function of graphene. For the case of a point charge, the induction energy is given by Eq. (46), in contrast to the corresponding results for metallic or insulating sheets $(53,50)$. The induction energy of a distant fixed dipole with graphene is given by Eq. (52), with a 2D metal by Eq. (53) and with a $2 \mathrm{D}$ insulator by Eq. (54)

In order to account correctly for the effects of the graphene bandstructure on the dispersoin (van der Waals) interactions of graphene, a theory must be used that is capable of describing long-ranged electron correlation physics without making the common assumption of pairwise additivity. Such a theory is the Random Phase Approximation (RPA), which can be carried though analytically when the interacting objects are far apart. Within the RPA, the dispersion (vdW) energy of a distant polarizable dipole with a 2D insulator, graphene, and a 2D metal are $E^{v d W} \propto-D^{-4}, E^{v d W} \propto-D^{-4} \ln \left(D / D_{0}\right)$ and $E^{v d W} \propto-D^{-3}$ respectively [18]. The vdW interaction energy per unit area between parallel graphene sheets separated by distance $D \rightarrow \infty$, within the RPA, is $E \propto-D^{-3}$ (see Eq. $(62,63)$ ), whereas the results 
for insulating and metallic $2 \mathrm{D}$ sheets are $E^{v d W} \propto-D^{-4}$ and $E^{v d W} \propto-D^{-2.5}$ respectively. For all the asymptotic geometries just described, pairwise additive vdW theories give the insulating results quoted above regardless of the electronic bandstructure of the sheet - i.e.the same power law $E^{v d W}=-c D^{-p}$ for insulators, semi-metals (graphene) or the $2 \mathrm{D}$ electron gas, with differences only in the numerical prefactor $c$.

The power laws described above set in only for rather large separations, and are not relevant near the equilibrium separation $D_{0}$ of the two objects. For closer separations analytic results canot be obtained because the physics of cohesion is no longer restriced to small-wavenumber response. One can nowadays nevertheless compute the RPA correlation energy, exact exchange energy and all other energy contributions within large bandstructure codes. For cases involving graphene, these are formidable calculations because of the need to sample the Brillouin zone very finely near the Dirac points where the $\pi_{z}$ and $\pi_{z}^{*}$ bands touch. Converged RPA results have nevertheless very recently been achieved for the layer stretching curve of graphite. These gave an equilibrium layer spacing $D_{0}$ and $C_{33}$ (interlayer bond stretching) elastic constant in good agreement with experiment, as well as predicting a layer binding energy of $48 \mathrm{meV} / \mathrm{C}$ atom, which constitutes a new benchmark in view of the widely varying experimental results.

The analytic work summarized here has predicted unusual forms for the distant asymptotic interactions of graphene with other structures. Perhaps it is now time to consider direct experimental confirmation using atomic Force Microscopy or vibrational spectroscopy with large flakes of graphene. Definitive experiments on the exfoliation, cleavage and layer binding energies of graphite would also be desirable.

\section{ACKNOWLEDGMENTS}

I thank T. Gould, A. White, A. Rubio, G. Kresse, G. Galli, W. Hanke, A. Savin, J. Angyan and I. Snook for useful discussions. Financial support was provided by a FAST grant from the Australian and French Governments, and an ARC Discovery Grant. 


\section{REFERENCES}

[2] A. H. Castro Neto, F. Guinea, N. M. R. Peres, K. S. Novoselov, and A. K. Geim, Rev. Mod. Phys. 81, 109 (2009).

[3] J. F. Dobson, A. White, and A. Rubio, Phys. Rev. Lett. 96, 073201 (2006).

[4] S. Lebegue, J. Harl, T. Gould, J. G. Angyan, G. Kresse, and J. F. Dobson, Phys. Rev. Lett. 105, 196401 (2010).

[5] R. Saito and M. Dresselhaus, Physical Properties of Carbon Nanotubes (Imperial College Press, London, 1998).

[6] X. Gonze, J.-M. Beuken, R. Caracas, F. Detraux, M. Fuchs, G.-M. Rignanese, L. Sindic, M. Verstraete, G. Zerah, F. Jollet, et al., Comp. Mater. Sci 25, 478 (2002).

[7] X. Gonze, G.-M. Rignanese, M. Verstraete, J.-M. Beuken, Y. Pouillon, R. Caracas, F. Jollet, M. Torrent, G. Zerah, M. Mikami, et al., Zeit. Kristallogr 220, 558 (2005).

[8] G. Kresse and J. Furthmueller, Phys. Rev. B 54, 11169 (1996).

[9] G. Kresse and D. Joubert, Phys. Rev. B 59, 1758 (1999).

[10] P. R. Wallace, Phys. Rev. 71, 622 (1947).

[11] W. R. Hanke, Phys. Rev. B 8, 4585 (1973).

[12] K. W. K. Shung, Phys. Rev. B 34, 979 (1986).

[13] I. E. Dzyaloshinskii, E. M. Lifshitz, and L. P. Pitaevskii, Adv. Phys. 10, 165 (1961).

[14] J. González, F. Guinea, and M. A. H. Vozmediano, Phys. Rev. B 63, 134421 (2001).

[15] S. Dassarma, Q. P. Li, and R. Joynt, Phys. Rev. B 45, 13713 (1992).

[16] W. Hanke and L. J. Sham, Phys. Rev. Lett. 33, 582 (1974).

[17] J. Gonzalez, F. Guinea, and M. A. H. Vozmediano, Phys. Rev. B 63, 134421 (2001).

[18] J. Ma, A. Michaelides, D. Alfe, G. Kresse, J. F. Dobson, A. Rubio, and E. Wang, unpublished (2010).

18 [19] J. F. Dobson, K. McLennan, A. Rubio, J. Wang, T. Gould, H. M. Le, and B. P. Dinte, Australian J. Chem. 54, 513 (2001). 
19 20] J. F. Dobson, J. Wang, B. P. Dinte, K. McLennan, and H. M. Le, Int. J. Quantum Chem. p. 579 (2005).

[21] O. Gunnarsson and B. I. Lundqvist, Phys. Rev. B 13, 4274 (1976).

[22] D. C. Langreth and J. P. Perdew, Sol. State Commun. 17, 1425 (1978).

[23] L. D. Landau and E. Lifshitz, Statistical Physics (Addison-Wesley, Reading, Massachusetts, 1969).

[24] J. Harris and A. Griffin, Phys. Rev. B 11, 3669 (1975).

[25] J. F. Dobson, Springer Lect. Notes Phys. 706, 443 (2006).

[26] M. Bostrom and B. E. Sernelius, Phys. Rev. B 61, 2204 (2000).

[27] B. E. Sernelius and P. Björk, Phys. Rev. B 57, 6592 (1998).

[28] L. A. Girifalco and R. A. Lad, J. Chem. Phys 25, 693 (1956).

[29] L. X. Benedict, N. G. Chopra, M. L. Cohen, A. Zettl, S. G. Louie, and V. H. Crespi, Chem. Phys. Lett. 286, 490 (1998).

[30] R. Zacharia, H. Ulbricht, and T. Hertel, Phys. Rev. B 69, 155406 (2004).

[31] D. C. Langreth, B. I. Lundqvist, and S. D. C.-K. et al., J. Phys. Cond. Matt 21, 084203 (2009).

[32] S. D. Chakarova-Kack, O. Borck, and E. S. et al., Phys. Rev. B 74, 155402 (2006).

[33] S. D. Chakarova-Kack, E. Schroder, and L. B. et al., Phys. Rev. Lett. 96, 146107 (2006).

[34] D. C. Langreth, M. Dion, and H. R. et al., Int. J. Quantum Chem. 101, 599 (2005).

[35] L. A. Girifalco, M. Hodak, and R. S. Lee, Phys. Rev. B 62, 13104 (2000).

[36] M. Dion, H. Rydberg, E. Schröder, D. C. Langreth, and B. I. Lundqvist, Phys. Rev. Lett. 92, 246401 (2004).

[37] H. Y. Kim, J. O. Sofo, D. Velegol, M. W. Cole, and A. A. Lucas, J. Chem. Phys. 124, 074504 (2006).

[38] A. White and J. F. Dobson, Phys. Rev. B 77, 075436 (2008).

[39] A. J. Misquitta, J. Spencer, A. J. Stone, and A. Alavi, Phys. Rev. B 82, 075312 (2010).

[40] J. F. Dobson, Dispersion (van der Waals) forces and time dependent density functional theory (Springer-Verlag, Berlin, 2006), p. 443. 
40 [41] A. Fetter and J. Walecka, Quantum Theory of Many-particle Systems (McGraw Hill, New York, 1971).

[42] A. Szabo and N. S. Ostlund, Modern Quantum Chemistry: Introduction to Advanced Electronic Structure Theory (McGraw Hill, New York, 1989).

[43] A. Gruneis, M. Marsman, J. Harl, L. Schimka, and G. Kresse, J. Chem. Phys. 131, 154115 (2009).

[44] J. G. Angyan, I. C. Gerber, A. Savin, and J. Toulouse, Phys. Rev. A 72, 012510 (2005).

[45] S. Kurth and J. P. Perdew, Phys. Rev. B 59, 10461 (1999).

[46] P. Mori-Sanchez, Aaron, and W. Yang, arXiv:0903.4403v1 (2009).

[47] J. Toulouse, I. C. Gerber, G. Jansen, A. Savin, and J. G. Angyan, Phys. Rev.Lett. 102, 096404 (2009).

[48] J. F. Dobson, in Topics in Condensed Matter Physics (Nova, New York, 1994).

[49] T. Miyake, F. Aryasetiawan, T. Kotani, M. van Schilfgaarde, M. Usuda, and K. Terakura, Phys. Rev. B 66, 245103 (2002).

[50] A. Marini, P. Garcia-Gonzalez, and A. Rubio, Phys. Rev. Lett. 96, 136404 (2006).

[51] J. Harl and G. Kresse, Phys. Rev. Lett. 103, 056401 (2009).

[52] T. Gould, K. Simpkins, and J. F. Dobson, Phys. Rev. B 77, 165134 (2008).

[53] T. Gould, E. M. Gray, and J. F. Dobson, Phys. Rev. B 79, 113402 (2009).

[54] J. Jung, P. Garcia-Gonzalez, J. F. Dobson, and R. W. Godby, Phys. Rev. B 70, 205107 (2004).

54 555 L. Spanu, S. Sorella, and G. Galli, Phys. Rev. Lett. 103, 196401 (2009).

[55] K. F. Allison, D. Borka, I. Radović, Lj. Hadžievski and Z. L. Mišković, Phys. Rev. B 80 (2009) 195405. 